\title{
Preparation of Schrödinger cat states of a cavity field via coupling to a superconducting charge qubit
}

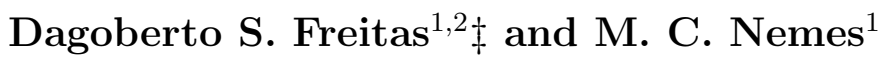 \\ 1 Departamento de Física, Instituto de Ciências Exatas, Universidade Federal de \\ Minas Gerais, 30123-970, Belo Horizonte, MG, Brazil \\ ${ }^{2}$ Departamento de Física, Universidade Estadual de Feira de Santana, 44036-900, \\ Feira de Santana, BA, Brazil \\ E-mail: dfreitas@uefs.br
}

\begin{abstract}
We extend the approach of Ref. 3] for preparing superposition states of a cavity field interacting with a superconducting charge qubit. We study effects of the nonlinearity on the creation of such states. We show that the main contribution of nonlinear effects is to shorten the time necessary to build the superposition.

PACS numbers: 42.50.Dv, 42.50.Ct, 74.50.+r
\end{abstract}

$\ddagger$ Author to whom any correspondence should be addressed.

Permanent address: Departamento de Física, Universidade Estadual de Feira de Santana, 44036-900, Feira de Santana, BA, Brazil. 


\section{Introduction}

The generation of nonclassical states of a radiation field has become increasingly important in the past years given its various applications in quantum communication. The feasibility of generating such nonclassical states has been established in several branches of physics such as cavity electrodynamics, trapped ions, quantum dots, atoms inside cavities and so on [1, 2]. In this contribution we discuss the issue in the context of a superconducting qubit in microcavity. It has been recently proposed a way to engineer quantum states using a SQUID charge qubit inside a cavity [3, 4] with a controllable interaction between the cavity field and the charge qubit. The key ingredients are a tunable gate voltage and a classical magnetic field applied to SQUID. In Ref. [3] a model is proposed including these ingredients and using some adequate approximations which allows for the linearization of the interaction and nonclassical states of the field are generated. In Ref. [4] the same model is used for the preparation of macroscopic quantum superposition states of a cavity field via coupling to a superconducting charge qubit.

We show that the essential contribution of nonlinear interaction is to shorten the time necessary to build the quantum state. Since decoherence is known to affect quantum effects uninterruptedly, they are working even when the quantum state is being formed. This has been studied and quantified in the context of cavity QED where it is shown that the more "quantum" is the superposition more rapid are the environmental effects during the process of creating the quantum state [5]. It is therefore interesting to envisage processes through which quantum superpositions are generated as fast as possible.

\section{The model}

We consider a system constituted by a SQUID type superconducting box with $n_{c}$ excess Cooper-pair charges connected to a superconducting loop via two identical Josephson junctions having capacitors $C_{J}$ and coupling energies $E_{J}$. An external control voltage $V_{g}$ couples to the box via a capacitor $C_{g}$. We also assume that the system operates in a regime consistent with most experiments involving charge qubits, in which only Cooper pairs coherently tunnel in the superconducting junctions. Therefore the system Hamiltonian may be written as [6]

$$
H_{q b}=4 E_{c h}\left(n_{c}-n_{g}\right)^{2}-2 E_{j} \cos \left(\frac{\pi \Phi_{X}}{\Phi_{0}}\right) \cos (\Theta),
$$

where $E_{c h}=e^{2} / 2\left(C_{g}+2 C_{J}\right)$ is the single-electron charging energy, $n_{g}=C_{g} V_{g} / 2 e$ is the dimensionless gate charge (controlled by $V_{g}$ ), $\Phi_{X}$ is the total flux through the SQUID loop and $\Phi_{0}$ the flux quantum. By adjusting the flux through the superconducting loop, one may control the Josephson coupling energy as well as switch on and off the qubitfield interaction. The phase $\Theta=\left(\phi_{1}+\phi_{2}\right) / 2$ is the quantum-mechanical conjugate of the number operator $n_{c}$ of the Cooper pairs in the box, where $\phi_{i}(\mathrm{i}=1,2)$ is the phase 
difference for each junction. The superconducting box is assumed to be working in the charging regime and the superconducting energy gap $\Delta$ is considered to be the largest energy involved. Moreover, the temperature $T$ is low enough so that the conditions $\Delta \gg E_{c h} \gg E_{J} \gg k_{B} T$, where $k_{B}$ is the Boltzmann constant. The superconducting box then becomes a two-level system with states $|g\rangle$ (for $\left.n_{c}=0\right)$ and $|e\rangle$ (for $\left.n_{c}=1\right)$ given that the gate voltage is near a degeneracy point $\left(n_{g}=1 / 2\right)$ [6] and the quasiparticle excitation is completely suppressed [7].

If the circuit is placed within a single-mode microwave superconducting cavity, the qubit can be coupled to both a classical magnetic field (generates a flux $\Phi_{c}$ ) and the quantized cavity field (generates a flux $\Phi_{q}=\eta a+\eta^{*} a^{\dagger}$, with $a$ and $a^{\dagger}$ the annihilation and creation operators), being the total flux through the SQUID given by $\Phi_{X}=\Phi_{c}+\Phi_{q}$ [8, 9, 10]. The parameter $\eta$ is related to the mode function of the cavity field. The system Hamiltonian will then read

$$
H=\hbar \omega a^{\dagger} a+E_{z} \sigma_{z}-E_{J}\left(\sigma_{+}+\sigma_{-}\right) \cos \left(\gamma I+\beta a+\beta^{*} a^{\dagger}\right),
$$

where we have defined the parameters $\gamma=\pi \Phi_{c} / \Phi_{0}$ and $\beta=\pi \eta / \Phi_{0}$. The first term corresponds to the free cavity field with frequency $\omega=4 E_{c h} / \hbar$ and the second one to the qubit having energy $E_{z}=-2 E_{c h}\left(1-2 n_{g}\right)$ with $\sigma_{z}=|e\rangle\langle e|-| g\rangle\langle g|$. The third term is the (nonlinear) photon-qubit interaction term which may be controlled by the classical flux $\Phi_{c}$. In general the Hamiltonian in equation (2) is linearized under some kind of assumption. In Ref. [3, for instance, the authors decomposed the cosine in Eq.(2) and expanded the terms $\sin \left[\pi(\eta a+H . c.) / \Phi_{0}\right]$ and $\cos \left[\pi(\eta a+H . c.) / \Phi_{0}\right]$ as power series in $a\left(a^{\dagger}\right)$. In this way, if the limit $|\beta| \ll 1$ is taken, only single-photon transition terms in the expansion are kept, and a Jaynes-Cummings type Hamiltonian (JCM) is then obtained. Here, in contrast to that, we adopt a similar technique to the one presented in reference [11] obtaining a JCM Hamiltonian valid for any value of $|\beta|$.

\section{Dynamics of the system}

The key idea of the method proposed in Ref. [11] which allows for the inclusion of nonlinear effects is the following: a unitary transformation is constructed in a way that diagonalizes the Hamiltonian leading it to a much simpler form. The nonlinear effects are therefore held in the transformation affecting directly the time evolution of the system in a tractable manner.

We first apply a unitary transformation to the full Hamiltonian given by (2) and make approximations afterwards. By applying the unitary transformation [1]

$$
\begin{aligned}
T & =\frac{1}{\sqrt{2}}\left\{-\frac{1}{2}\left[D^{\dagger}(\alpha, \gamma)-D(\alpha, \gamma)\right] I-\frac{1}{2}\left[D^{\dagger}(\alpha, \gamma)+D(\alpha, \gamma)\right] \sigma_{z}\right. \\
& \left.+D(\alpha, \gamma) \sigma_{+}+D^{\dagger}(\alpha, \gamma) \sigma_{-}\right\}
\end{aligned}
$$

to the Hamiltonian in equation (2), with $D(\alpha, \gamma)=D(\alpha) e^{i \frac{\gamma}{2}}$ where $D(\alpha)=\exp \left[\left(\alpha a^{\dagger}-\right.\right.$ $\left.\alpha^{*} a\right)$ ] is the Glauber's displacement operator, with $\alpha=i \beta^{*} / 2$, we obtain the following 
transformed Hamiltonian

$$
\begin{aligned}
H_{T} & \equiv T H T^{\dagger} \\
& =\hbar \omega a^{\dagger} a+\frac{E_{J}}{2} \sigma_{z}+i \frac{\hbar}{2}\left[\omega\left(\beta a-\beta^{*} a^{\dagger}\right)+2 i \frac{E_{z}}{\hbar}\right] \sigma_{x} \\
& +\frac{E_{J}}{2} \cos \left[2\left(\beta a+\beta^{*} a^{\dagger}\right)+2 \gamma\right] \sigma_{z} \\
& -i \frac{E_{J}}{2} \sin \left[2\left(\beta a+\beta^{*} a^{\dagger}\right)+2 \gamma\right]\left(\sigma_{+}-\sigma_{-}\right)+\left|\frac{\beta}{2}\right|^{2} .
\end{aligned}
$$

This result holds for any value of the parameter $\beta$. For the regime in which $\hbar \omega|\beta| \gg E_{J}$, that can be obtained for $|\beta| \geq 0.25$, the Hamiltonian in Eq.(41) becomes

$$
H_{T} \cong \hbar \omega a^{\dagger} a+i \frac{\hbar \omega}{2}\left[\left(\beta a-\beta^{*} a^{\dagger}\right)+2 i \frac{E_{z}}{\hbar \omega}\right] \sigma_{x}
$$

Our Hamiltonian in Eq.(5) becomes a Jaynes-Cummings type Hamiltonian. The term $\left|\frac{\beta}{2}\right|^{2}$ was not taken into account because it just represents an overall phase. Now we are interested in the generation of Schrödinger cat states (SC) in the above system. The time evolution of the state vector for an initial state $|\psi(0)\rangle$ is

$$
\begin{aligned}
|\psi(t)\rangle & =T^{\dagger} U_{T}(t) T|\psi(0)\rangle \\
& =U(t)|\psi(0)\rangle,
\end{aligned}
$$

where $U_{T}(t)=\exp \left(-i H_{T} t / \hbar\right)$ is the time evolution operator in the transformation representation and $U(t)=T^{\dagger} U_{T}(t) T$ is the time evolution operator in the original representation that is given by

$$
U(t)=T^{\dagger} \exp \left\{-i\left[\omega a^{\dagger} a+i \frac{\omega}{2}\left[\left(\beta a-\beta^{*} a^{\dagger}\right)+2 i \frac{E_{z}}{\hbar \omega}\right] \sigma_{x}\right] t\right\} T .
$$

Using the identity $e^{A+B}=e^{A} e^{B} e^{-[A, B] / 2}$ the equation (6]) may be written in the form

$$
U(t)=T^{\dagger} e^{-i \omega a^{\dagger} a t} e^{-\frac{\omega \beta}{2}\left(a^{\dagger}-a\right) \sigma_{x} t} e^{-\frac{\omega^{2} \beta}{4}\left(a^{\dagger}+a\right) \sigma_{x} t^{2}} e^{i \frac{E_{z}}{\hbar} \sigma_{x} t} T
$$

where for simplicity we consider $\beta$ as real. After some algebra, the time evolution operation in the original representation becomes

$$
U(t)=e^{-i \omega a^{\dagger} a t}\left(\begin{array}{cc}
D(\tilde{\beta}) e^{-i \frac{E_{z}}{\hbar} t} & 0 \\
0 & D^{\dagger}(\tilde{\beta}) e^{i \frac{E_{z}}{\hbar} t}
\end{array}\right)
$$

where we define the parameter $\tilde{\beta}=i \frac{\omega^{2} \beta}{4} t^{2}$ and $D(\tilde{\beta})$ is the Glauber's displacement operator as defined above.

Considering the state vector as having the following initial condition

$$
|\psi(0)\rangle=|0\rangle\left[\frac{1}{\sqrt{2}}(|e\rangle+|g\rangle)\right],
$$

or the qubit prepared in a superposition states of $|e\rangle$ and $|g\rangle$ and the field in a vacuum state $|0\rangle$, the time evolution of the state vector is given by

$$
\begin{aligned}
|\psi(t)\rangle & =U(t)|\psi(0)\rangle \\
& =\frac{1}{\sqrt{2}}\left[e^{-i \frac{E_{z}}{\hbar} t}\left|e^{-i \omega t} \tilde{\beta}\right\rangle|e\rangle+e^{i \frac{E_{z}}{\hbar} t}\left|-e^{-i \omega t} \tilde{\beta}\right\rangle|g\rangle\right] .
\end{aligned}
$$


Now we rotate the qubit so that $|e\rangle \rightarrow \frac{1}{\sqrt{2}}(|e\rangle-|g\rangle)$ and $|g\rangle \rightarrow \frac{1}{\sqrt{2}}(|e\rangle+|g\rangle)$. This rotation is equivalent to applying the operator $R=\frac{1}{\sqrt{2}}\left[1+\left(\sigma_{+}-\sigma_{+}\right)\right]$on the states $|e\rangle$ and $|g\rangle$. Applying $R$ on the state given by equation (10) a superposition of coherent states will be obtained

$$
R|\psi(t)\rangle=\frac{1}{\sqrt{2}}\left(\Phi_{+}|e\rangle-\Phi_{-}|g\rangle\right)
$$

with the SC

$$
\Phi_{ \pm}=\frac{1}{\sqrt{2}}\left[e^{-i \frac{E_{z}}{\hbar} t}\left|e^{-i \omega t} \tilde{\beta}\right\rangle|e\rangle \pm e^{i \frac{E_{z}}{\hbar} t}\left|-e^{-i \omega t} \tilde{\beta}\right\rangle|g\rangle\right]
$$

The result is an entangled state involving qubit and a cavity field. If one measures the charge state (either in $|g\rangle$ or $|e\rangle$ ), that action will collapse the $R|\psi(t)\rangle$ into a SC state $\Phi_{ \pm}$. The form of Eq.(11) is very similar to the SC obtained in Ref. 4. But, in contrast to that, we did not do use the condition $|\beta| \ll 1$. In our scheme, as $|\beta|$ is large, and the value of the amplitude of coherent states are proportional to $t^{2}$, the time for preparing an observable SC state is much shorter than that in other schemes. The approach used here is similar to the approach used in Ref. [12] for the preparation of SC with cold ions beyond the Lamb-Dicke limit.

\section{Conclusion}

In conclusion, we have presented an approach for preparing SC states of mode of cavity field interacting with a superconducting charge qubit. In contrast to others schemes we include nonlinear effects. In general, approximations are made directly to the full Hamiltonian in equation (2) neglecting all higher orders of $|\beta|$. In our scheme, we first apply an unitary transformation to the Hamiltonian (2) and make the relevant approximations after performing the transformation. The result obtained holds for any value of the parameter $\beta$. In the regime in which $\hbar \omega|\beta| \gg E_{J}$, that can be obtained for $|\beta| \geq 0.25$, the Hamiltonian becomes a Jaynes-Cummings type Hamiltonian. Based on the measurement of charge states, we show that SC states of a single-mode cavity field can be generated. Here, as $|\beta|$ is large, and the amplitude of the coherent states are proportional to $t^{2}$, the time for preparing observable $\mathbf{S C}$ states is much shorter than in the linear regimes.

\section{Acknowledgments}

D.S.F and M.C.N acknowledge the financial support from Conselho Nacional de Desenvolvimento Cientifico e Tecnológico - CNPq (150232/2012-8), Brazil.

\section{References}

[1] M. Brune, E. Hagley, J. Dreyer, X. Matre, A. Maali, C. Wunderlich, J. M. Raimond, and S. Haroche, Phys. Rev. Lett. 77, 4887 (1996).

[2] C. Monroe, D. M. Meekhof, B. E. King, and D. J. Wineland, Science 272, 1131 (1996). 
[3] Yu-xi Liu, L. F. Wei, and F. Nori, Europhys. Lett. 67, 941 (2004).

[4] Yu-xi Liu, L. F. Wei, and F. Nori, Phys. Rev. A 71, 063820 (2005).

[5] J. G. Peixoto, M. C. Nemes, Journal of Optics. B 5, 265 (2002).

[6] Yu. Makhlin, G. Schn, and A. Shnirman, Rev. Mod. Phys. 73, 357 (2001).

[7] D. V. Averin, and Y. V. Nazarov, Phys. Rev. Lett. 69, 1993 (1992).

[8] J. Q. You and F. Nori, Phys. Rev. B 68, 064509 (2003).

[9] W. A. Al-Saidi and D. Stroud, Phys. Rev. B 65, 224512 (2002).

[10] Shi-Liang Zhu, Z. D. Wang and Kaiyu Yang, Phys. Rev. A 68, 034303 (2003).

[11] H. Moya-Cessa, A. Vidiella-Barranco, J. A. Roversi, D. S. Freitas and S. M. Dutra, Phys. Rev. A 59, 2518 (1999).

[12] M. Feng, Phys. Lett. A, 282, 230 (2001). 